\title{
Vibration monitoring using fibre optic sensors in a lead-bismuth eutectic cooled nuclear fuel assembly
}

\author{
Ben De Pauw ${ }^{1,2,3 *}$, Alfredo Lamberti ${ }^{1}$, Julien Ertveldt ${ }^{1}$, Ali Rezeyat ${ }^{1}$, Katrien Van Tichelen ${ }^{2}$, \\ Steve Vanlanduit ${ }^{1}$, Francis Berghmans ${ }^{3}$ \\ ${ }^{1}$ Acoustics and vibration research group, Vrije Universiteit Brussel, Pleinlaan 2, Elsene, Belgium; \\ E-Mails: bdepauw@vub.ac.be; allamber@vub.ac.be; julien.ertveldt@vub.ac.be; arezayat@vub.ac.be; \\ svlandui@vub.ac.be \\ ${ }^{2}$ Belgian Nuclear Research Centre (SCK•CEN), Boeretang 200, Mol, Belgium; E-Mails: \\ kvtichel@sckcen.be \\ ${ }^{3}$ Brussels Photonics Team (B-Phot), Vrije Universiteit Brussel, Pleinlaan 2, Elsene, Belgium; E-Mails: \\ fberghma@vub.ac.be \\ * Author to whom correspondence should be addressed; E-Mail: bdepauw@vub.ac.be; \\ Tel.: +3226292324.
}

Published: 10 November 2015

\begin{abstract}
Excessive fuel assembly vibrations in nuclear reactor cores should be avoided in order not to decrease the lifetime of the assembly and in order to prevent the occurrence of safety hazards. This issue is particularly relevant to new reactor designs that use liquid metals, such as for example a molten lead-bismuth eutectic, as a coolant. The flow of molten heavy metal around and through the fuel assembly may cause the latter to vibrate and hence to suffer degradation as a result of fretting wear. In this paper we demonstrate the use of optical fibre sensors to measure the vibration induced by the coolant flow in a lead-bismuth eutectic cooled installation and we apply modal analysis techniques to derive modal parameters of the system. We show that the vibration on the fuel rods can be experimentally measured with minimal intrusiveness on the fuel pin owing to the small dimensions and fundamental properties of the optical fibres. With these sensors, we determine the vibration amplitude and modal parameters of the fuel assembly containing the fuel rods during different steps of the operation of the facility, including the initial start-up of the coolant circulation as well as during continuous operation. This first-of-a-kind experiment also demonstrates the potential of optical fibre based instrumentation in these harsh environments.
\end{abstract}


Keywords: liquid metal flows; FBG; vibration; modal analysis; experiments; fluid-structure interaction

\section{Introduction}

This paper pertains to the research activities conducted with the aim to develop MYRRHA, which is a prototype nuclear reactor belonging to the so-called fourth generation [1]. MYRRHA will be an accelerator driven reactor cooled with molten lead-bismuth eutectic (LBE). As in any nuclear reactor, the vibration levels of reactor internals have to be kept within certain limits. Excessive vibrations of reactor components have caused several component failures in the past [2]. One particularly important component is the fuel assembly. In MYRRHA, one fuel assembly consists of a total of 127 wire spaced parallel fuel rods. Figure 1 illustrates a sub-assembly consisting of 7 fuel rods. These fuel rods are placed vertically in the reactor and are mechanically supported only at the bottom. The top remains unsupported. The LBE coolant is flowing upwards and interacts with the fuel assembly. This fluid-structure interaction causes the fuel assembly to vibrate. A wire spacer is wrapped around every fuel rod to ensure that they remain properly spaced during operation. The spacing between the individual fuel pins is limited to a couple of millimetres, which leaves little room to install vibration sensors. Moreover and in order to obtain reliable vibration readings, the sensors should not affect the flow of LBE. Therefore the selected sensors should have very small dimensions.

Different optical and electro-mechanical vibration sensors or techniques have already been successfully applied to measure the coolant-flow induced vibration in a nuclear fuel assembly [3-5]. Heavy liquid metal coolants have a relatively high density, flow at elevated temperature and are corrosive and opaque. This shortens the list of potentially adequate sensors or techniques. For our application, we needed a vibration measuring technique that can operate reliably for a sufficiently long time in a flow of $\mathrm{LBE}$ at $300^{\circ} \mathrm{C}$. To that end, we selected fibre optic Bragg grating (FBG) based sensors which we have validated for this specific environment as described in [6]. In this validation process we demonstrated that we can improve the lifetime during which the FBGs are dependable vibration sensors whilst subjected to $\mathrm{LBE}$ at $300^{\circ} \mathrm{C}$ to beyond 700 hours.

In this paper we demonstrate actual vibration measurements of a LBE cooled fuel assembly. The goal of the experiments was first to evaluate the operation of the fibre sensors for vibration measurements in a mock-up LBE cooled loop. To do so, we instrumented a 7-pin scaled mock-up of the MYRRHA fuel assembly and subjected it to a flow of LBE. The second objective was to demonstrate successful evaluation of the vibration amplitudes and identification of the modal parameters in several LBE flow conditions. Note that the goal is to determine the vibrations in a mock-up situation, with the eventual intention to support the optimization of the design of the fuel assembly. To the best of our knowledge, FBG sensors have never been applied for this purpose. Consequently the effects of nuclear radiation do not need to be taken into account in this study and the influences of radioactivity on the flow-induced vibration can be considered to be a secondary effect. 


\section{Experimental set-up}

A FBG consists of a periodic variation of the refractive index of the core of an optical fibre and is fabricated using dedicated laser-based techniques (see also [7]). The FBG is characterized by a resonance wavelength at which it reflects light, referred to as Bragg wavelength $\lambda_{B}$. This $\lambda_{B}$ depends on the strain and on the temperature applied to the FBG. Thus, tracing the value of $\lambda_{B}$ as a function of time allows using the FBG as a sensor. Since the expected wavelength fluctuation is small and signal degradation due to the environment is expected to occur (as explained in detail in [6]), we opted to use a phase-correlation based approach to analyse the acquired signals [8]. This approach allowed achieving the best trade-off between signal-to-noise ratio and calculation speed even if the FBG spectra become distorted. In this fashion, we were able to record Bragg wavelength shifts as small as $0.25 \mathrm{pm}$ corresponding to a local strain level difference of about $0.2 \mu \epsilon$. In the end spectra were recorded and analysed in real-time with an acquisition rate of almost 5000 measurements per second.

Every fuel pin of the 7-pin assembly was instrumented with an optical fibre. Each fibre contained 8 FBGs uniformly distributed over the fuel pin length as also illustrated in Figure 1. This fuel assembly was then inserted in a set-up that could circulate LBE whilst keeping the LBE temperature constant and uniform. This allowed us, combined with the high-pass filtered explained later, to remove the influence of temperature on the FBGs.
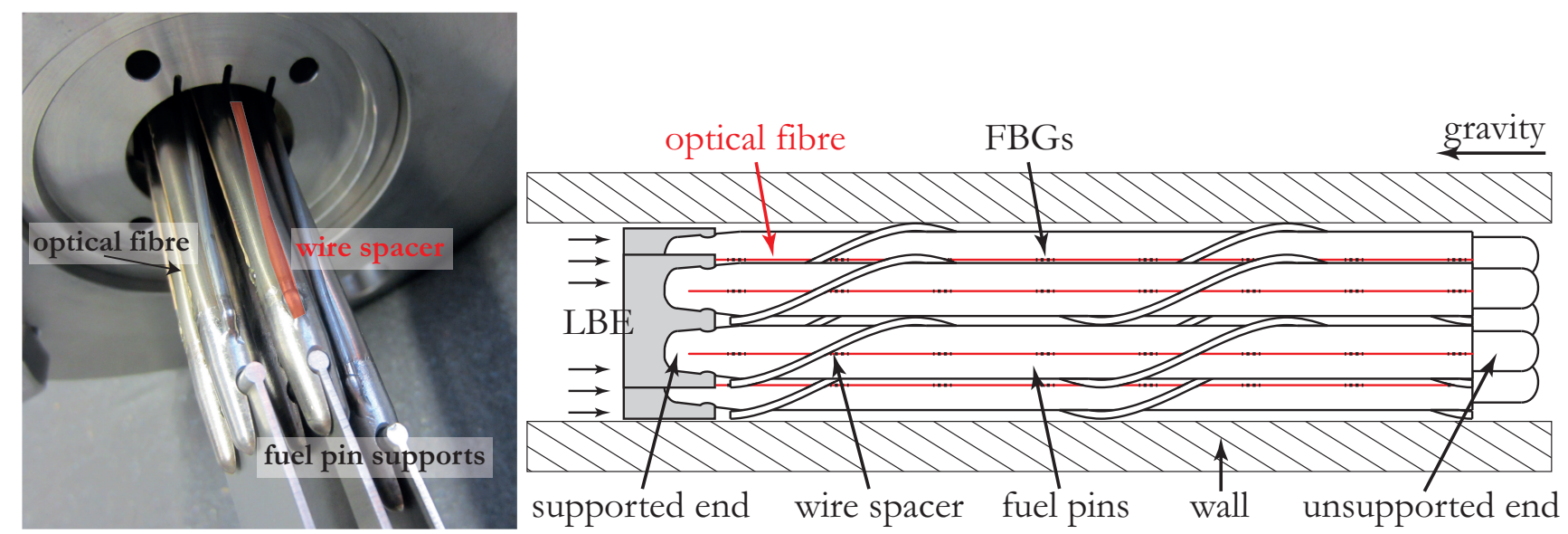

Figure 1. Concept drawings and photograph of the 7-pin fuel assembly.

\section{Results and Discussion}

\subsection{Vibration amplitude evaluation}

Many different operational conditions can occur in the coolant flow of a fuel assembly including different coolant flow velocities and transients during start-up. All these operational conditions should be investigated when studying the flow-induced vibration. We measured the dynamic strain using the FBGs on the surface of the fuel pins in steady flow and varying flow conditions as well as during transients e.g. when the pump is switched on. The procedure was as follows. We started from stagnant LBE and we gradually increased the flow velocity up to nearly $6 \mathrm{~m} / \mathrm{s}$. Once the maximum flow velocity was reached, we stopped the pump. We illustrate the course of the procedure in Figure 2 with from top to bottom the 
LBE flow velocity, the average strain values as measured with all the FBGs on all fuel pins and these measurements with a high-pass filter applied to determine the instantaneous vibration amplitude. This high-pass filtering consisted of subtracting Savitzky-Golay filtered data from the unfiltered data with a span of 20000 samples i.e. 4 seconds of measurement time. The largest vibration amplitude clearly occurred during the start-up of the pump. Note that the operation of the pump starts at approximately $0.6 \mathrm{~m} / \mathrm{s}$, generating a transient in the flow velocity. In this case, the vibration amplitude exceeded $70 \mu \epsilon$. However, this transient disappears after a few seconds. We believe that the transient during the start-up of the pump is caused by the initial push of the flow on the fuel assembly resulting in a compressive force (see Figure 2 (middle)). When increasing the flow velocity we observed a corresponding increase in the vibration amplitude and drag. The drag corresponds to the 'DC' value (i.e. with a low-pass filter applied) whilst the vibration amplitude corresponds to the 'AC' value (i.e. with a high-pass filter applied) of the unfiltered strain data. For LBE coolant flow velocities in MYRRHA (indicated with the red line), we observed an average vibration amplitude close to $0.5 \mu \epsilon$. At the highest tested flow velocity, we measure an instantaneous vibration amplitude of nearly $3 \mu \epsilon$. When the highest flow speed is reached, we stopped the pump. A control loop managing the pump prevents the occurrence of excessively fast transients in the pump rotation and hence the shut-down was not instantaneous.

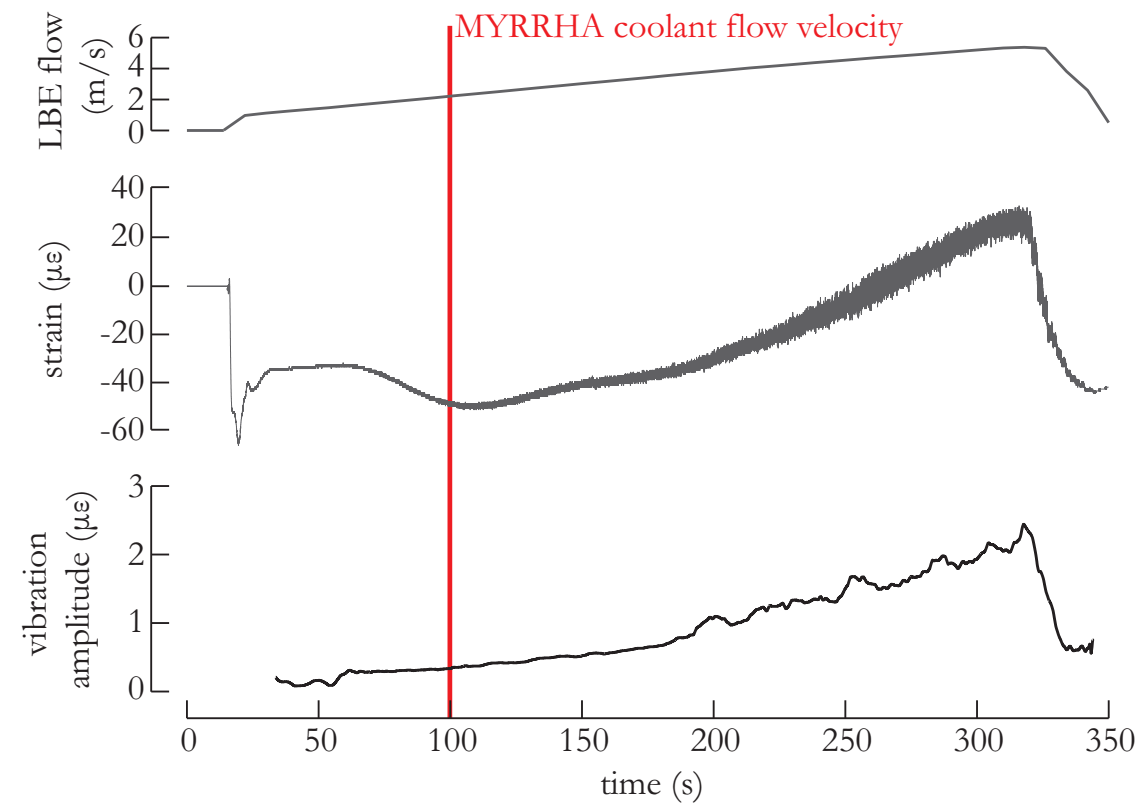

Figure 2. top: rotation speed of the pump during the experiment which corresponds to the LBE flow velocity; middle: corresponding average strain values as measured with all the FBGs on all fuel pins; bottom: high-pass filter applied to the strain values of the middle figure as this corresponds to the instantaneous vibration amplitude and as this eliminate residual influence of temperature on the FBGs.

\subsection{Modal analysis results}

To characterize the vibrations of the fuel pins in the fuel assembly, we used the raw, unfiltered dynamic strain measurements of the FBG sensors as input for a modal analysis. We followed the methodology explained in [9] to identify the modal characteristics of the vibration. The methodology relies on a 
state-of-the-art operational modal estimator. Using this methodology, we were able to identify the multiple eigen-modes corresponding to certain flexural modes. This multitude of eigen-modes for each flexural mode is explained by the complex intra fuel pin interaction that is present in the fuel assembly. We obtained the resulting spread of eigen-frequencies and corresponding damping ratios for the first 4 flexural modes as displayed in Figure 3. As can be seen from the figures, the mode shapes deviate somewhat from the classical beam modes for a cantilevered beam. This is mainly the result of clustering the fuel pins in a very tightly packed manner. Near the fixation, located leftmost in the figures, the strain amplitude is highest in most cases.

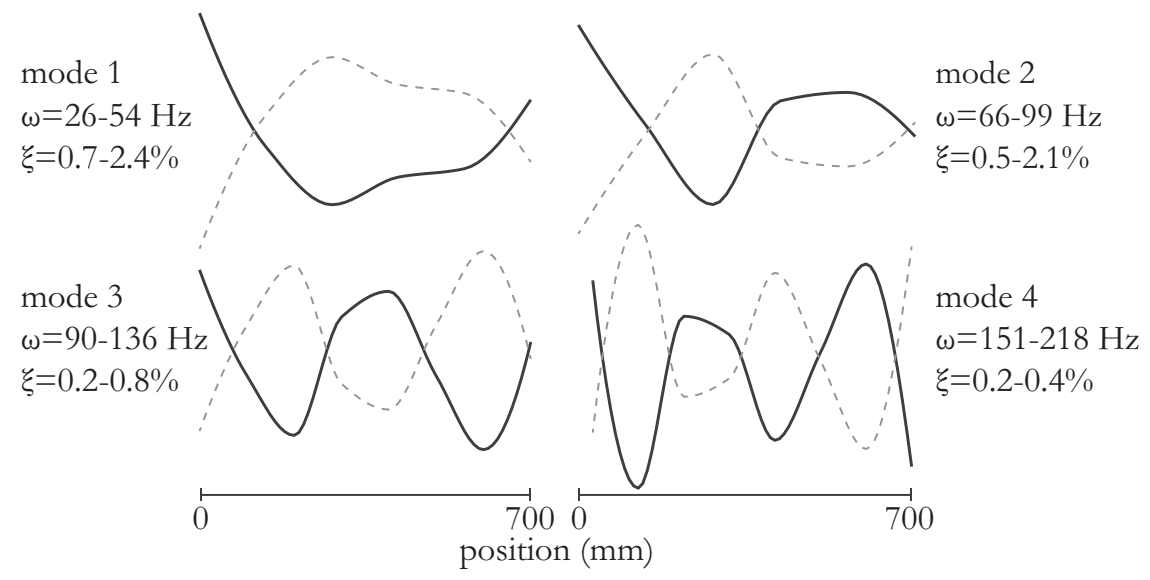

Figure 3. Experimentally estimated mode shapes and approximate eigen-frequency and damping ranges during external excitation of the fuel pins.

\section{Conclusions}

We have demonstrated in this paper for the first time to the best of our knowledge, the successful use of optical fibre Bragg grating (FBG) based sensors for vibration measurements in a lead-bismuth eutectic (LBE) cooled installation. We applied FBGs to determine the vibration induced by the coolant flow in a LBE cooled nuclear fuel assembly. To illustrate this, we first evaluated the vibration amplitude of the fuel assembly during different phases of operation. We then extended our study and used the measured small vibrations to identify modal characteristics of the fuel assembly during operation.

With these results, we have established a set of tools required to experimentally characterize liquid metal cooled fuel assembly vibrations in nuclear reactor cores that allow to avoid excessive vibration that could lead to a decreased lifetime of the assembly and the occurrence of safety hazards. We focused on measuring the vibration of the individual fuel rods in the fuel assembly, but the technique can also be applied to other components or sections of the installation.

\section{Acknowledgments}

The authors gratefully acknowledge the support of the Belgian Nuclear Research Centre (SCK• CEN). This work was also partially supported by the IWT-SBO Project with Contract 110070 'e-SHM with AM', and the Methusalem and Hercules Foundations Flanders. The Belgian Science Policy Interuniversity Attraction Pole P7/35 is acknowledged as well. 


\section{Conflicts of Interest}

The authors declare no conflict of interest.

\section{References}

1. H. Ait Abderrahim, P. Baeten, D. De Bruyn, and R. Fernandez. MYRRHA - A multi-purpose fast spectrum research reactor. Energy Conversion and Management, 63:4-10, November 2012.

2. R. D. Blevins. Flow-induced vibration in nuclear reactors: a review. Progress in Nuclear Energy, 4:25-49, 1979.

3. S. S. Chen. Parallel-flow-induced vibration of fuel rods. Nuclear Engineering and Design, 18:253-278, 1972.

4. V. Prakash, M. Thirumalai, R. Prabhakar, and G. Vaidyanathan. Assessment of flow induced vibration in a sodium-sodium heat exchanger. Nuclear Engineering and Design, 239(1):169-179, January 2009.

5. M. J. Pettigrew. The behaviour of weldable strain gauges under nuclear reactor core conditions. Nuclear Engineering and Design, 263:350-361, October 2013.

6. B. De Pauw, A. Lamberti, A. Rezayat, J. Ertveldt, S. Vanlanduit, K. Van Tichelen, T. Geernaert, and F. Berghmans. Signal-to-noise ratio evaluation of fibre Bragg gratings for dynamic strain sensing at elevated temperatures in a liquid metal environment. Journal of Lightwave Technology, 33(12):1-8, 2015.

7. A. Othonos and K. Kalli. Fiber Bragg gratings: Fundamentals and Application in Telecommunications and Sensing. Artech House, London, 1999.

8. A. Lamberti, S. Vanlanduit, B. De Pauw, and F. Berghmans. Influence of Fiber Bragg Grating Spectrum Degradation on the Performance of Sensor Interrogation Algorithms. Sensors, pages 24258-24277, 2014.

9. B. De Pauw, W. Weijtjens, S. Vanlanduit, K. Van Tichelen, and F. Berghmans. Operational modal analysis of flow-induced vibration of nuclear fuel rods in a turbulent axial flow. Nuclear Engineering and Design, 284:19-26, 2015.

(c) 2015 by the authors; licensee MDPI, Basel, Switzerland. This article is an open access article distributed under the terms and conditions of the Creative Commons Attribution license (http://creativecommons.org/licenses/by/4.0/). 